\title{
CoreGRID/ERCIM Workshop on Grids, Clouds and P2P Computing - CGWS2011
}

\author{
Marco Danelutto ${ }^{1}$, Frédéric Desprez ${ }^{2}$, Vladimir Getov ${ }^{3}$, and Wolfgang Ziegler ${ }^{4}$ \\ 1 Univ. of Pisa (I) \\ 2 INRIA and ENS Lyon (F) \\ 3 Univ. of Westminster (UK) \\ ${ }^{4}$ Fraunhofer SCAI (D)
}

CoreGRID is a European research Network of Excellence (NoE) that was initiated in 2004 as part of the EU FP6 research framework and run up to 2008. CoreGRID partners, from 44 different countries, developed theoretical foundations and software infrastructures for large-scale, distributed Grid and P2P applications. An ERCIM sponsored CoreGRID Working Group was established to ensure the continuity of the CoreGrid programme after the official end of NoE. The working group extended its interests to include the emerging field of (service based) cloud computing which is of great importance to the European software industry. Its main goals consist in i) sustaining the operation of the CoreGRID Network, ii) establishing a forum to encourage collaboration between the Grid and P2P Computing research communities, and (iii) encourage research on the role of cloud computing as a new paradigm for distributed computing in e-Science.

In particular, the ERCIM CoreGRID working group managed to organize an annual CoreGRID workshop, traditionally associated to the Euro-Par conference, thus continuing the tradition of the CoreGRID workshops regularly run once per year during the NoE activities. Past ERCIM CoreGRID workshops have been organized in Delft (2009) and Ischia-Naples (2010). In 2011 the workshop has been organized in Bordeaux. As usual the topics of interest included Service Level Agreements, Data \& Knowledge Management, Scheduling, Virtual environments, Network monitoring, Volunteer Computing Systems, Trust \& Security, Self-* and adaptive mechanisms, Advanced programming models, IaaS, PaaS and SaaS, Tools and Environments for Application Development and Execution.

The 2011 ERCIM CoreGRID workshop gathered around 40 researchers from the european community, on August 29, 2011. Nine papers were presented after one keynote talk.

The keynote talk was given by Franoise Baude (Univ. de Nice, CNRS, INRIA Sophia Antipolis) about an overview of the Grid Component Model (GCM) that allows large applications to be ported in a seamless way over Grids and now Clouds.

The first paper presented a study of the mapping of evolving applications over resource managers. Results showed that resource usage and application response 
time can be significantly improved with short scheduling times. The second paper discussed the use of model checking to support conflict resolution in multiple non-functional concern management. A trade-off must be found between the ability to develop independently management of the individual concerns and the detection and resolution of conflicts that may arise when combining the independently developed management code. The next paper showed how consistent reconfiguration protocols can be derived for stream-based ASSISTANT applications, and their costs was characterized in terms of proper performance models. The fourth paper described challenges around highly interactive virtual environments, also known as Real-Time Online Interactive Applications (ROIA). A dynamic resource management system RTF-RMS which implements a loadbalancing strategy for ROIA on Clouds was presented. In the fifth paper, the architecture of Contrail federations of Clouds were presented and motivated. Beside supporting user authentication and applications deployment, Contrail federations aim at providing extended SLA management functionalities, by integrating the SLA management approach of SLA@SOI project in the federation architecture. The sixth paper presented a novel tool to synthesize ontologies for the Abstract Grid Workflow Language (AGWL). Experiments based on two separate application domains were shown that demonstrate the effectiveness of our approach by semi-automatically generating ontologies which are then used to automatically create workflow applications. The seventh paper presented the chemical machine, an interpreter for the Higher Order Chemical Language. The design follows that of logic/functional languages and bridges the gap between the highly abstract chemical model and the physical machine by an abstract interpreter engine. The next paper described OP2, an "active" library framework for the solution of unstructured mesh applications. The OP2 code generation and compiler framework which, given an application written using the OP2 API, generates efficient code for state-of-the-art hardware (e.g. GPUs and multi-core CPUs) were discussed. The last paper proposed a dynamic load balancing strategy to enhance the performance of parallel association rule mining algorithms in the context of a Grid computing environment.

We wish to thank all those that contributed to the success of the workshop: authors submitting papers, invited speakers, colleagues participating in the refereeing process / discussion sessions and Euro-Par 2011 organizers whose invaluable support greatly helped in the organisation of the Workshop. 